IISc-CTS-10/01

hep-ph/0104286

\title{
Higgs Mass in the Standard Model from Coupling Constant Reduction
}

\author{
B. Ananthanarayan \\ J. Pasupathy \\ Centre for Theoretical Studies, \\ Indian Institute of Science, \\ Bangalore 560 012, India.
}

\begin{abstract}
Plausible interrelations between parameters of the standard model are studied. The empirical value of the top quark mass, when used in the renormalization group equations, suggests that the ratio of the colour $\mathrm{SU}(3)$ gauge coupling $g_{3}$, and the top coupling $g_{t}$ is independent of the renormalization scale. On the other hand, variety of top-condensate models suggest that the Higgs self-coupling $\lambda$ is proportional to $g_{t}^{2}$. Invoking the requirement that the ratio $\lambda(t) / g_{t}^{2}(t)$ is independent of the renormalization scale $t$, fixes the Higgs mass. The pole mass of the Higgs [which differs from the renormalization group mass by a few percent] is found to be $\sim 154 \mathrm{GeV}$ for the one-loop equations and $\sim 148 \mathrm{GeV}$ for the two-loop equations.
\end{abstract}

PACS number(s): 12.15.-y, 11.10.Hi, 14.80.Bn

Typeset using REVTEX 


\section{INTRODUCTION}

It is widely believed that not all the parameters occurring in the standard model are independent and there must be some interrelation between them. The most striking feature of the standard model in this regard is the fact that top quark mass is nearly same as the electroweak symmetry breaking parameter $(\mathrm{EWSB}), m_{t} \simeq 174 \mathrm{GeV}\left(=\left(2 \sqrt{2} G_{F}\right)^{-1 / 2}\right.$, where $G_{F}$ is the Fermi constant). This has lead several authors to propose mechanisms similar to the BCS superconductors for EWSB [1]. It was already noted way back in 1961 by Nambu and Jona-Lasinio [2], that in these models, besides the zero mass collective excitations, [which become the longitudinal component of the gauge bosons] there is also a scalar bound state of the massive quasi-fermions with a mass appproximately twice that of the fermion mass. This theoretical prediction was confirmed experimentally in BCS superconductors in Raman spectra studies. This generic relation between the fermion mass and the associated scalar particles is present in He-III and in spectra of several nuclei where pairing place an important role. It is conceivable, that BCS type symmetry breaking is truly ubiquitous and extends also to electroweak symmetry breaking. For our purposes it is sufficient to note that such theories the scalar self-coupling is proportional to the square of the quasi-fermion scalar coupling.

Bounds on the top-quark mass as well as the Higgs mass were obtained many years ago by Cabibbo et. al. [3] by requiring that the coupling constants remain positive and the vacuum state be stable. This in turn has given rise to a large activity which uses phenomenological ideas like infrared fixed points, quasi-fixed points and supersymmetric extension of these. For a review of these topics, see e.g., ref. [4].

On the other hand several years ago Zimmermann introduced the idea of reduction of coupling constants in a renormalizable field theory [5]. It is conceivable that a theory written in terms of several coupling constants $\lambda_{0}, \lambda_{1}, \ldots, \lambda_{n}$ contains really only one independent constant $\lambda_{0}$ with others expressible as functions of $\lambda_{0}$,

$$
\lambda_{j}=\lambda_{j}\left(\lambda_{0}\right), \quad j=1, \ldots, n .
$$

Zimmermann, then showed that the beta functions must satisfy relations of the type:

$$
\beta_{0} \frac{d \lambda_{j}}{d \lambda_{0}}=\beta_{j}
$$

where $\beta_{j}$ denoted the $\beta$-function corresponding to $\lambda_{j}$.

Assuming a power series expansion, one may try to solve these equations and then discover relations between couplings leading to a reduction in the number of independent ones. In the context of the standard model this programme has been considered in detail in ref. [6]. A major difficulty in this programme is the following. If one considers for example the reduction of the $\mathrm{U}(1)$ gauge coupling $g_{1}$ in terms of the $\mathrm{SU}(3)$ colour coupling $g_{3}$ in the lowest order, it has no solution, which can be traced to the fact that while non-abelian gauge couplings have negative beta funnction (asymptotically free), in contrast to U(1) which has positive beta function with a coupling that grows with energy.

The renormalization group equations being first order differential equations require for their solution the specification of the couplings at some arbitrary scale. In the one-loop approximation, the equations for $g_{1}, g_{2}$ and $g_{3}$ the gauge couplings, and $g_{t}$, the Yukawa 
coupling of the Higgs to the top quark, can be solved easily using the experimental values of the gauge couplings say at the Z-mass and the top quark mass. The details are provided in Sec. II. In this introduction we simply note from Fig. 1 where the solution of the coupling constants $g_{3}^{2}(t)$ and $g_{t}^{2}(t)$ for an energy scale running from $10 \mathrm{GeV}$ to a $\mathrm{TeV}$ is displayed, that $g_{t}^{2}(t)$ like $g_{3}^{2}(t)$ also falls with increasing energy and the ratio of $g_{t}^{2}(t) / g_{3}^{2}(t)$ roughly remains the same over this range. This is discussed in more detail later.

It was mentioned above that BCS type theories that the Higgs self-coupling $\lambda$ is proportional $g_{t}^{2}$. To carry this analogy over to a relativistic field theory, we need to specify the energy scale where we expect these relations to hold.

Guided by the observation of the near scale independence of the ratio of the $g_{3}^{2}(t) / g_{t}^{2}(t)$ made above, we now make a simple hypothesis. The ratio $\lambda(t) / g_{t}^{2}(t)$ is independent of the renormailzation scale $t$. This fixes the integration constant of renormalization group equation for $\lambda$ and therefore the Higgs mass.

In an earlier work, one of us had used this criterion of scale independence of the coupling constant ratios to one-loop to determine the Higgs mass [0]. Here we extend these considerations to two-loop renormalization group equations. In ref. [7], the difference between the pole mass and the renormalization group mass was ignored. Here we repair the deficiency. The rest of this paper is organised as follows. In Sec. II we specify our normalisations, the pole mass and renormalization group mass relations and the solution of renormalization group equations in the one-loop and two-loop cases. In Sec. III, we compare our results with indirect estimates of the Higgs mass from electroweak precision data and also some of the other theoretical ideas on the subject. An appendix explains the relation of our assumption of scale independence to the coupling constant reduction method.

\section{RENORMALIZATION GROUP EQUATIONS AND RESULTS}

In a renormalizable field theory, one first specifies the values of the various couplings and masses at some arbitrary momentum scale $\mu$. The physical matrix elements are obtained from the Green functions. The requirement that the physical quantities are independent of $\mu$ leads to the renormalization group equations.

The renormalization group equations up to two-loops in perturbation theory have been calculated in the $\overline{\mathrm{MS}}$ renormalization scheme for the couplings of the standard model and its minimal supersymmetric extension, for a review, see e.g. ref. [4. In the following we shall consider the solution of the renormalization group equations for the standard model setting all couplings other than $g_{1}, g_{2}, g_{3}, g_{t}$ and $\lambda$, to zero. The renormalization group equations valid for $\mu>m_{t}$ read $\left(t=\ln \left(\mu / m_{t}\right)\right)$ :

$$
\begin{aligned}
& \frac{\mathrm{d} g_{1}^{2}}{\mathrm{~d} t}=\frac{g_{1}^{4}}{8 \pi^{2}}\left(\frac{41}{10}+\frac{1}{16 \pi^{2}}\left(\frac{199}{50} g_{1}^{2}+\frac{27}{10} g_{2}^{2}+\frac{44}{5} g_{3}^{2}-\frac{17}{10} g_{t}^{2}\right)\right) \\
& \frac{\mathrm{d} g_{2}^{2}}{\mathrm{~d} t}=\frac{g_{2}^{4}}{8 \pi^{2}}\left(-\frac{19}{6}+\frac{1}{16 \pi^{2}}\left(\frac{9}{10} g_{1}^{2}+\frac{35}{6} g_{2}^{2}+12 g_{3}^{2}-\frac{3}{2} g_{t}^{2}\right)\right) \\
& \frac{\mathrm{d} g_{3}^{2}}{\mathrm{~d} t}=\frac{g_{3}^{4}}{8 \pi^{2}}\left(-7+\frac{1}{16 \pi^{2}}\left(\frac{11}{10} g_{1}^{2}+\frac{9}{2} g_{2}^{2}-26 g_{3}^{2}-2 g_{t}^{2}\right)\right)
\end{aligned}
$$




$$
\begin{aligned}
\frac{\mathrm{d} g_{t}^{2}}{\mathrm{~d} t}= & \frac{g_{t}^{2}}{8 \pi^{2}}\left(-\frac{17}{20} g_{1}^{2}-\frac{9}{4} g_{2}^{2}-8 g_{3}^{2}+\frac{9}{2} g_{t}^{2}\right. \\
& +\frac{1}{16 \pi^{2}}\left(\frac{1187}{600} g_{1}^{4}-\frac{9}{20} g_{1}^{2} g_{2}^{2}-\frac{23}{4} g_{2}^{4}+\frac{19}{15} g_{1}^{2} g_{3}^{2}+9 g_{2}^{2} g_{3}^{2}-108 g_{3}^{4}+\frac{393}{80} g_{1}^{2} g_{t}^{2}\right. \\
& \left.\left.+\frac{225}{16} g_{2}^{2} g_{t}^{2}+36 g_{3}^{2} g_{t}^{2}-12 g_{t}^{4}-12 g_{t}^{2} \lambda+6 \lambda^{2}\right)\right) \\
\frac{\mathrm{d} \lambda}{\mathrm{d} t}= & \frac{1}{16 \pi^{2}}\left(\frac{27}{200} g_{1}^{4}+\frac{9}{20} g_{1}^{2} g_{2}^{2}+\frac{9}{8} g_{2}^{4}-\frac{9}{5} g_{1}^{2} \lambda-9 g_{2}^{2} \lambda-6 g_{t}{ }^{4}+12 g_{t}^{2} \lambda+24 \lambda^{2}\right. \\
& +\frac{1}{16 \pi^{2}}\left(-\frac{3411}{2000} g_{1}^{6}-\frac{1677}{400} g_{1}^{4} g_{2}^{2}-\frac{289}{80} g_{1}^{2} g_{2}^{4}+\frac{305}{16} g_{2}^{6}-\frac{171}{100} g_{1}^{4} g_{t}^{2}\right. \\
& +\frac{63}{10} g_{1}^{2} g_{2}^{2} g_{t}^{2}-\frac{9}{4} g_{2}^{4} g_{t}^{2}+\frac{1887}{200} g_{1}^{4} \lambda+\frac{117}{20} g_{1}^{2} g_{2}^{2} \lambda-\frac{73}{8} g_{2}^{4} \lambda-\frac{8}{5} g_{1}^{2} g_{t}{ }^{4}-32 g_{3}^{2} g_{t}{ }^{4} \\
& +\frac{17}{2} g_{1}^{2} g_{t}^{2} \lambda+\frac{45}{2} g_{2}^{2} g_{t}^{2} \lambda+80 g_{3}^{2} g_{t}^{2} \lambda+\frac{108}{5} g_{1}^{2} \lambda^{2}+108 g_{2}^{2} \lambda^{2} \\
& \left.\left.+30 g_{t}{ }^{6}-3 g_{t}{ }^{4} \lambda-144 g_{t}^{2} \lambda^{2}-312 \lambda^{3}\right)\right)
\end{aligned}
$$

Here we solve these coupled differential equations both for the one-loop as well as the two-loop case. We have proceeded as follows: we first take the values of the gauge couplings $g_{1}, g_{2}$ and $g_{3}$ at the Z-peak and evolve them via the renormalization group equations to the scale $m_{t}$, with 5 flavors of quarks. For scales larger than $m_{t}$ we employ the equations with 6 quark flavors. The values we take at the Z-peak are $g_{1}=0.462, g_{2}=0.652, g_{3}=1.221$. We take the pole mass for the top-quark to be $174 \mathrm{GeV}$. The relation between between the pole mass $m_{t}^{\text {pole }}$ and the renormalization group mass $m_{t}(\mu)$ at the scale $\mu$ is

$$
m_{t}(\mu)=m_{t}^{\text {pole }}\left(1+\delta_{t}(\mu)\right)
$$

with $m_{t}(\mu)=(v / \sqrt{2}) g_{t}(\mu), v=246 \mathrm{GeV}$. The correction term $\delta_{t}(\mu)$ is described in detail in ref. [4]. When computed numerically we find it to be small and for the case at hand, $\delta_{t}\left(\mu=m_{t}\right) \simeq-0.05$, which in turn corresponds to a value of $g_{t}\left(\mu=m_{t}\right)=0.95$.

With these inputs it is now easy to obtain solutions for $g_{1}, g_{2}, g_{3}$ and $g_{t}$. It was mentioned in the introduction that both $g_{3}$ and $g_{t}$ decrease asymptotically and their ratio remains a constant. This is displayed in Fig. 1, where the functional dependence of $g_{3}^{2}(t)$ and $g_{t}^{2}(t)$ in the interval corresponding to $\mu=10 \mathrm{GeV}$ to $1 \mathrm{TeV}$ is displayed. It is sufficient to note here that in this interval while both $g_{3}$ and $g_{t}$ drop by a factor of nearly 2 , their ratio approximately remains constant. Later on we also study the behaviour of this ratio for higher energy intervals in the one-loop and two-loop cases. It will be seen that the constancy improves at the two-loop level as compared to the one-loop level.

Now, to determine $\lambda$ we proceed as follows. Consider an arbitrary value at the scale $\mu=m_{t}$ for $\lambda(0)$. Given this the corresponding value of $\lambda(t)$ is fixed from the renormalization group equation for $\lambda(t)$, in the entire domain of $t$ where the equations are valid.

We solve these equations numerically and consider the ratio $R(t)=\lambda(t) / g_{t}^{2}(t)$ conveniently normalized to unity at $t=0: R(t) / R(0)$. The results of the computations are 
plotted for values of $\mu$ between $m_{t}$ and a large scale, say $10^{10} \mathrm{GeV}$, which then corresponds to $t: 0<t<17.87$ in Fig. 2. We find that the value of $\lambda(0)=0.176$ corresponding to the middle line is the one that best satisfies our criterion of constancy of $R(t)$. The behaviour of $R(t)$ when $\lambda(0)$ is varied from slightly from 0.176 is also shown in Fig. 2. Clearly even such small departures of $\lambda(0)$ from 0.176 lead to significant $t$ dependence and the corresponding ratio of couplings fail to meet our criterion of scale independence. Larger variations would lead to even larger departure from the desired constancy. It should be stressed here that the best value for $\lambda(0)$ determined by the scale independence criterion does not depend significantly on the range of $t$.

Starting from this best value $\lambda(0)=0.176$, the corresponding $\lambda(t)$ is used to determine the Higgs mass as follows. First the renormalization group mass is found from the equation

$$
m_{H}(\mu)=\sqrt{2 \lambda(\mu)} v
$$

The pole mass is related to the renormalization group equation mass above by

$$
m_{H}(\mu)=m_{H}^{\text {pole }}\left(1+\delta_{H}(\mu)\right) .
$$

Details of $\delta_{H}(\mu)$ are given in the review, ref. [4]. Numerically again it is small and we find it to be $\simeq-0.05$, for $\mu=m_{H}$. Using this, we find that at one-loop

$$
m_{H}^{\text {pole }}=154 \mathrm{GeV}(\text { one }- \text { loop}) .
$$

Comparing this value of $154 \mathrm{GeV}$ to the value given in ref. [7] of $160 \mathrm{GeV}$, the small difference arises due to the following improvements. Here we have taken into account the pole mass of the top-quark to be $174 \mathrm{GeV}$ unlike the earlier work where it was the renormalization group mass. In the earlier work the $t=0$ corresponded to $\mu=m_{Z}$ and the fact that only five flavours are operative below $m_{t}$ was ignored. Furthermore, the value for the Higgs mass quoted here corresponds to its pole mass, which differs by about $5 \%$ from its renormalization group mass.

In Fig. 3, the analogous computation is performed with the two-loop beta functions. The best value of $\lambda(0)$ changes slightly to 0.162 . Repeating the calculations described above for the pole mass, we find at two-loop

$$
m_{H}^{\text {pole }}=148 \mathrm{GeV}(\text { two }- \text { loops }) .
$$

We now return to the ratio of $R(t)=g_{3}^{2}(t) / g_{t}^{2}(t)$ which we observed in the introduction to be nearly constant. We plot this ratio, again normalized to its value at $t=0$ for the range of values for $\mu$ from $m_{t}$ to $10^{10} \mathrm{GeV}$, in Fig. 4. It is seen that at one-loop level that this is nearly constant, with the ratio tending to increase slightly for increasing energies. In Fig. 5 we show the results for the same ratio at the two-loop level. It is observed that the ratio is unity to within as little as $5 \%$ even at energies as high as $10^{10} \mathrm{GeV}$. Note that this feature is virtually independent of the Higgs mass when varied around our preferred value of $148 \mathrm{GeV}$ by a few percent on either side and the figures corresponding to these variations in the Higgs mass lie on top of each other (see caption of Fig. 5).

This leads to the question whether one can "determine" the top mass by the requirement of scale independence of the ratio $g_{t}^{2}(t) / g_{3}^{2}(t)$. In order to address this, instead of considering 
a variety of values for the top-quark pole mass and the corresponding changes in $g_{t}(\mu)$, we can simply illustrate this equivalently by varying the values of $g_{t}$ at $\mu=174 \mathrm{GeV}$. We have chosen the values $0.85,0.95$ and 1.05. It is seen from Fig. 6 that at one-loop level the scale independence criterion would put the top-quark mass at a slightly lower value than the experimental one. On the other hand, from Fig. 7 we see that at the two-loop level this criterion leads to a value for the top-quark mass quite close to the experimental value, when $m_{H}$ is in the neighbourhood of $148 \mathrm{GeV}$.

It is thus seen that our criterion of scale independence of the ratios of couplings has an internal consistency. At the one-loop level $g_{3}(t)$ and $g_{t}(t)$ are independent of $\lambda$ and the ratio $g_{3}^{2}(t) / g_{t}^{2}(t)$ is not quite constant. However, remarkably at the two-loop level both the ratios $\lambda(t) / g_{t}^{2}(t)$ and $g_{3}^{2}(t) / g_{t}^{2}(t)$ maintain scale independence over a significant range of energies.

\section{DISCUSSION}

Turning now to experiments, one has bounds on the Higgs mass from precision electroweak data on the W-mass, $\sin ^{2} \theta_{W}$, and leptonic width of $\mathrm{Z}$, as recently reviewed by Marciano [8]:

$$
\begin{gathered}
m_{H}=53_{-40}^{+77} \mathrm{GeV}\left(\text { from } m_{W}\right) \\
m_{H}=67_{-27}^{+45} \mathrm{GeV}\left(\text { from } \sin ^{2} \theta_{\mathrm{W}}\right) \\
m_{H}=208_{-180}^{+340} \mathrm{GeV}\left(\text { from } Z \rightarrow l^{+} l^{-}(\gamma)\right)
\end{gathered}
$$

One also has the LEPII bound

$$
m_{H}>106 \mathrm{GeV}
$$

On the other hand a Baysean analysis combining the data from direct and indirect searches for the Higgs has been done by D'Agostini and Degrassi [9], which leads them to expect Higgs mass to be around $160-170 \mathrm{GeV}$ with an uncertainty of about 50 to $60 \mathrm{GeV}$.

In the present work we have not tried to incorporate, the standard model in a more encompassing theory, several of which have been proposed over the last three decades.

Composite models (for a review, see e.g. ref. [10]) tend to suggest a larger Higgs mass than the values obtained in the present work. While it is true that the Higgs mechanism for explaining spontaneous symmetry breaking was introduced in analogy with condensed matter physics, it is conceivable that there may be other settings. There are many geometrical ideas in the subject which have been proposed in the last three decades. Here we shall limit ourselves to brief comments about some results which are phenomenologically interesting.

For example in a version of noncommutative geometry, considered by Okumura [11] the Higgs field along with the gauge field appears as a connection on $M_{4} \times Z_{N}$, where $M_{4}$ is the usual spacetime manifold and $Z_{N}$ is a discrete space. Okumura obtains the coupling constant relation at the unification scale

$$
g_{2}^{2}=g_{1}^{2}=4 \lambda \neq g_{3}^{2} .
$$

In view of the last inequality there is no grand unification in this model. Extrapolating eq. (13) to lower energies, yields the result 


$$
m_{H} \simeq 158 \mathrm{GeV}
$$

for the renormalization group mass.

In another class of theories considered for example by Fairlie [12] and by Manton [13], the Higgs field makes its appearance from dimensional reduction. A gauge field which should transform as an anti-symmetric tensor under $S O(N-1,1),(N>4)$ when reduced with respect to the Lorentz group $S O(3,1)$ can contain Lorentz scalars just as a Lorentz four vector when reduced with respect to rotations contains a three vector and a three scalar. Reducing for example a gauge field theory in 6 dimensions with internal symmetry of the graded Lie group $S U(2 \mid 1)$ Fairlie found $\sin ^{2} \theta_{W}=1 / 4$ and a Higgs mass of $426 \mathrm{GeV}$. In these models, the Higgs self-coupling arises in a manner similar to the quartic coupling of the gauge field from invariance requirements in higher dimensions.

In view of the fact that superstring theories imply higher dimensional theories, these ideas in which Higgs makes its presence due to reduction of gauge connections in higher dimensions may be worth re-examining.

Yet another class of interesting theories is due to Roepstroff and Vehns [14]. In these theories, the Higgs plays the mathematical role of a super-connection which is an extension of the idea of a usual connection for a gauge field on space-time manifolds. Here again, relations between gauge couplings $g_{1}, g_{2}$ and $\lambda$ are obtained. For example, in the absence of fermions the model predicts $m_{H}=2 m_{Z}$, whereas a refined version which includes fermions as well yields a value of $m_{H} \approx 160 \mathrm{GeV}$.

Of course, currently most of the phenomenological activity is centered around the minimal supersymmetric extension of the standard model. While tree level relation puts the lightest Higgs to be below the Z-mass, radiative corrections puts it at a higher value. If one solves the standard model renormalization group equations at two-loops as we have done here, and identifies the Higgs of the standard model with the lightest Higgs of the minimal supersymmetric standard model, one may wish to explore plausible values of the supersymmetry scale $M_{S U S Y}$ as follows. Given $m_{H}, \lambda$ is determined as a function of $t$ and one may want to find out at what value of $t=\ln \left(M_{S U S Y} / m_{t}\right)$ the tree-level relation:

$$
\lambda\left(M_{S U S Y}\right)=\frac{1}{8}\left(\frac{3}{5} g_{1}^{2}\left(M_{S U S Y}\right)+g_{2}^{2}\left(M_{S U S Y}\right)\right) \cos ^{2} 2 \beta
$$

is satisfied. If the Higgs mass is varied from $115-155 \mathrm{GeV}$ we find (setting $\cos ^{2} 2 \beta=1$ ), that $M_{S U S Y}$ varies from something of order a $\mathrm{TeV}$ at the lower mass end, to a few thousand $\mathrm{TeV}$ at the higher end. It would be interesting to carry out a detailed analysis including the effects of the full supersymmetric spectrum.

In summary, it is interesting to note that the scale independence hypothesis leads us to expect a Higgs mass of about $150 \mathrm{GeV}$, and this value is quite consistent with the limits obtained from precision electro-weak data. Experimental discovery of the Higgs and the determination of its mass at the Tevatron in this range remains a distinct possibility. Should the Higgs turn out to have a mass of around $150 \mathrm{GeV}$, it would strongly support the scale independence of the coupling constant ratios.

Acknowledgements: JP thanks J. D. Bjorken, T. Ferbel and V. Srinivasan for their encouragement and valuable correspondence. 


\section{Appendix}

The following is a brief summary of the work of W. Zimmermann and collaborators. Details can be found in ref. [5.6].

Consider a renormalizable field theory involving coupling constants $\lambda_{0}, \lambda_{1}, \ldots \lambda_{n}$. A functional relation between coupling constants can arise when there is a symmetry in the Lagrangian or even otherwise. Assume now that all couplings are asymptotically free, i.e., $\lambda_{0}, \lambda_{1}, \ldots, \lambda_{n}$ tend to zero as the renormalization scale tends to infinity, and fundamental relations of the type

$$
\lambda_{i}=\lambda_{i}\left(\lambda_{0}\right) i=1,2, \ldots n
$$

make it possible to express the $\lambda_{i}(i=1, \ldots n)$ in terms of $\lambda_{0}$. Consider any arbitrary Green function of the theory. One may study the renormalization group transformation property of the Green function first in terms of the original theory where $\lambda_{0}, \lambda_{1}, \ldots \lambda_{n}$ are all regarded as independent and the reduced theory written in terms of $\lambda_{0}$. Comparing the two, consistency demands the relation

$$
\beta_{\lambda_{0}} \frac{d \lambda_{i}}{d \lambda_{0}}=\beta_{\lambda_{i}}
$$

between the beta functions of the theory.

A number of interesting relations following from eqns. (A.1) and (A.2) have been studied in ref. [6]. In the context of the standard model however one runs into difficulty, since $g_{1}^{2}$ the coupling corresponding to $U(1)$ groups is asymptotically increasing. In fact, consider a reduction program of $g_{1}^{2}$ in terms of $g_{3}^{2}$ in a power series

$$
g_{1}^{2}=C_{0} g_{3}^{2}+C_{1} g_{3}^{4}+\ldots
$$

For three generations the renormalization group equations for $g_{1}^{2}$ and $g_{3}^{2}$ read in the one-loop approximation as

$$
\begin{aligned}
& \frac{d g_{1}^{2}}{d t}=\frac{g_{1}^{4}}{8 \pi^{2}}\left(\frac{41}{40}\right) \\
& \frac{d g_{3}^{2}}{d t}=\frac{g_{3}^{2}}{8 \pi^{2}}(-7) .
\end{aligned}
$$

Using $(A .3),(A .4)$ and $(A .5)$ in $(A .2)$ finds for the coefficient $C_{0}$

$$
C_{0}^{2} \frac{41}{10}=-7 C_{0}
$$

which has solutions (a) $C_{0}=0$; trivial, or (b) $C_{0}=-70 / 41$; negative and therefore unacceptable.

This difficulty is circumvented in ref. [6] by discarding the reductions hypothesis for $g_{1}$ and in view of its smallness in relation to $g_{3}$ the top coupling $g_{t}$ and the Higgs self-coupling $\lambda$ incorporate it as a perturbation on other reduction equations. These procedures lead to very low values for the top-quark mass. Details can be found for example in ref. [6]. 
It is interesting to compare the reduction hypothesis, eq. (A.2) in relation to our approach in this paper where we have required the criterion:

$$
\frac{d}{d t}\left(\lambda(t) / g_{t}^{2}(t)\right)=0
$$

it follows from eq. $(A .7)$

$$
\beta_{\lambda} \frac{g_{t}^{2}(t)}{\lambda(t)}=\beta_{g_{t}} .
$$

Comparing eq. (A.8) with eq. (A.2) we see that our criterion of scale independence, $(A .7)$ is consistent with the reduction hypothesis if

$$
\frac{d g_{t}^{2}}{d \lambda}=\frac{g_{t}^{2}}{\lambda}
$$

Clearly if $(A .7)$ is valid, then $(A .9)$ is valid as well.

Thus our scale independence condition is consistent with the reduction hypothesis but is not demanded by it. On the other hand, we have seen in Fig. 7 that the ratio of the strong coupling $g_{3}$ and the coupling $g_{t}$ is scale independent and in fact could be used to determine the top-quark mass within its experimental limits. It is therefore conceivable that the Higgs coupling is also following a similar pattern. 


\section{REFERENCES}

[1] W. A. Bardeen, C. T. Hill and M. Lindner, Phys. Rev. D 41, 1647 (1990); Y. Nambu in New Theories in Physics, Proceedings of the XI International Symposium on Elementary Particle Physics, Kazmimierz, Poland 1988, reprinted in "Broken symmetry: Selected papers of Y. Nambu," T. Eguchi and K. Nishijima, eds., Singapore, Singapore: World Scientific (1995) 467 p. (World Scientific series in 20th century physics. 13); V. A. Miransky, M. Tanabashi and K. Yamawaki, Phys. Lett. B 221, 177 (1989).

[2] Y. Nambu and G. Jona-Lasinio, Phys. Rev. 122, 345 (1961), reprinted in T. Eguchi and K. Nishijima [1].

[3] N. Cabibbo, L. Maiani, G. Parisi and R. Petronzio, Nucl. Phys. B 158, 295 (1979).

[4] B. Schrempp and M. Wimmer, Prog. Part. Nucl. Phys. 37, 1 (1996) hep-ph/9606386.

[5] W. Zimmermann, Commun. Math. Phys. 97, 211 (1985).

[6] J. Kubo, K. Sibold and W. Zimmermann, Nucl. Phys. B 259, 331 (1985), Phys. Lett. B 220, 185 (1989); Phys. Lett. B 220, 191 (1989), see also J. Kubo, M. Mondragon, N. D. Tracas and G. Zoupanos, Phys. Lett. B 342, 155 (1995) hep-th/9409003].

[7] J. Pasupathy, Mod. Phys. Lett. A 15, 1605 (2000) hep-ph/0006258].

[8] W. J. Marciano, hep-ph/0003181.

[9] G. D'Agostini and G. Degrassi, hep-ph/0001269.

[10] R. S. Chivukula, hep-ph/0011264.

[11] Y. Okumura, Eur. Phys. J. C 4, 711 (1998) hep-ph/9707350].

[12] D. B. Fairlie, Phys. Lett. B 82, 97 (1979).

[13] N. S. Manton, Nucl. Phys. B 158, 141 (1979).

[14] G. Roepstorff and C. Vehns, hep-ph/0006065. 


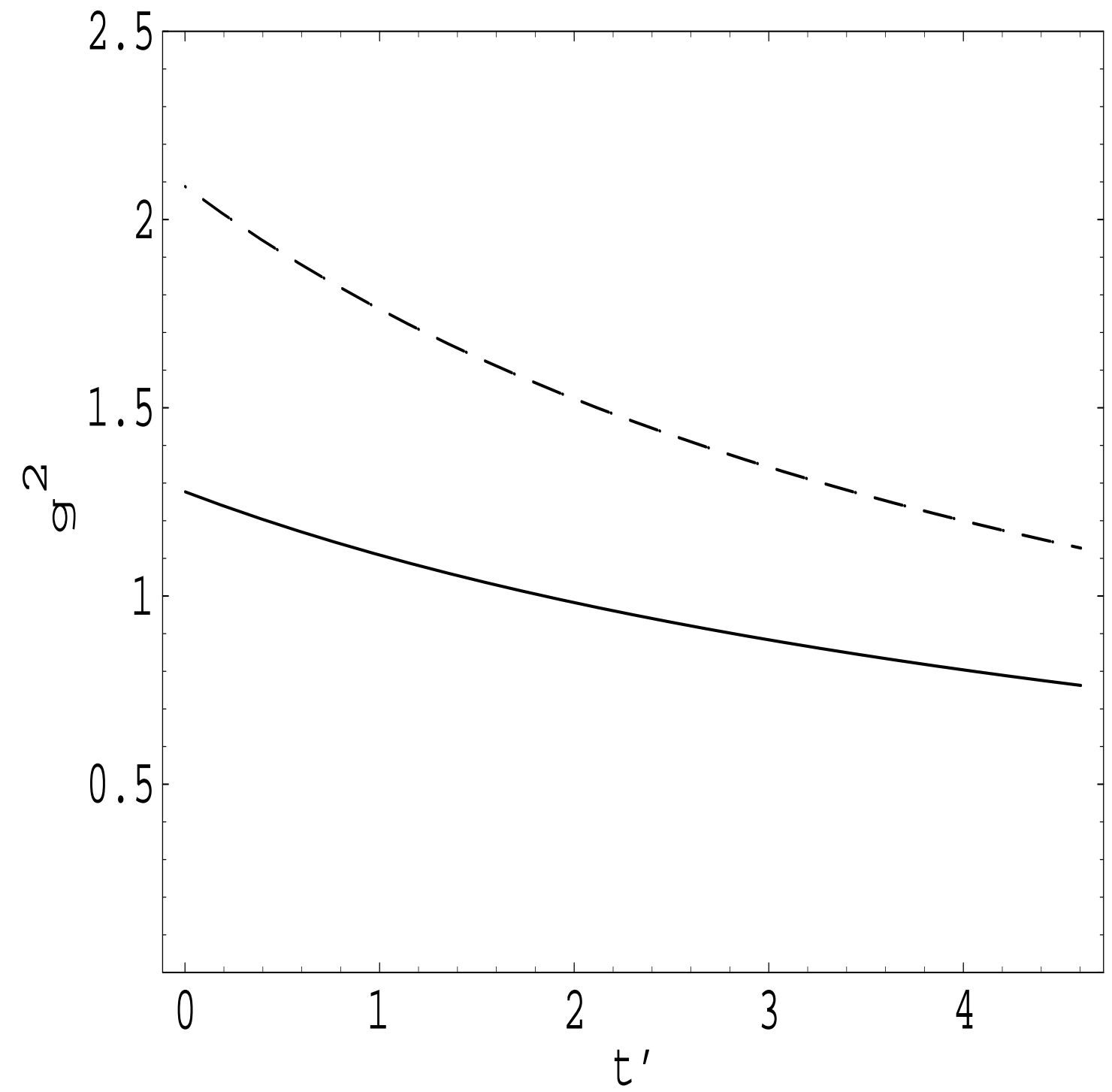

FIG. 1. Plot of $g_{t}^{2}$ and $g_{3}^{2}, t^{\prime}=\ln (\mu / 10)$ at one-loop (solid and dashed respectively), corresponding to $\alpha_{S}\left(M_{Z}\right)=0.119, m_{t}^{\text {pole }}=174 \mathrm{GeV}$, see Sec. II for details. 


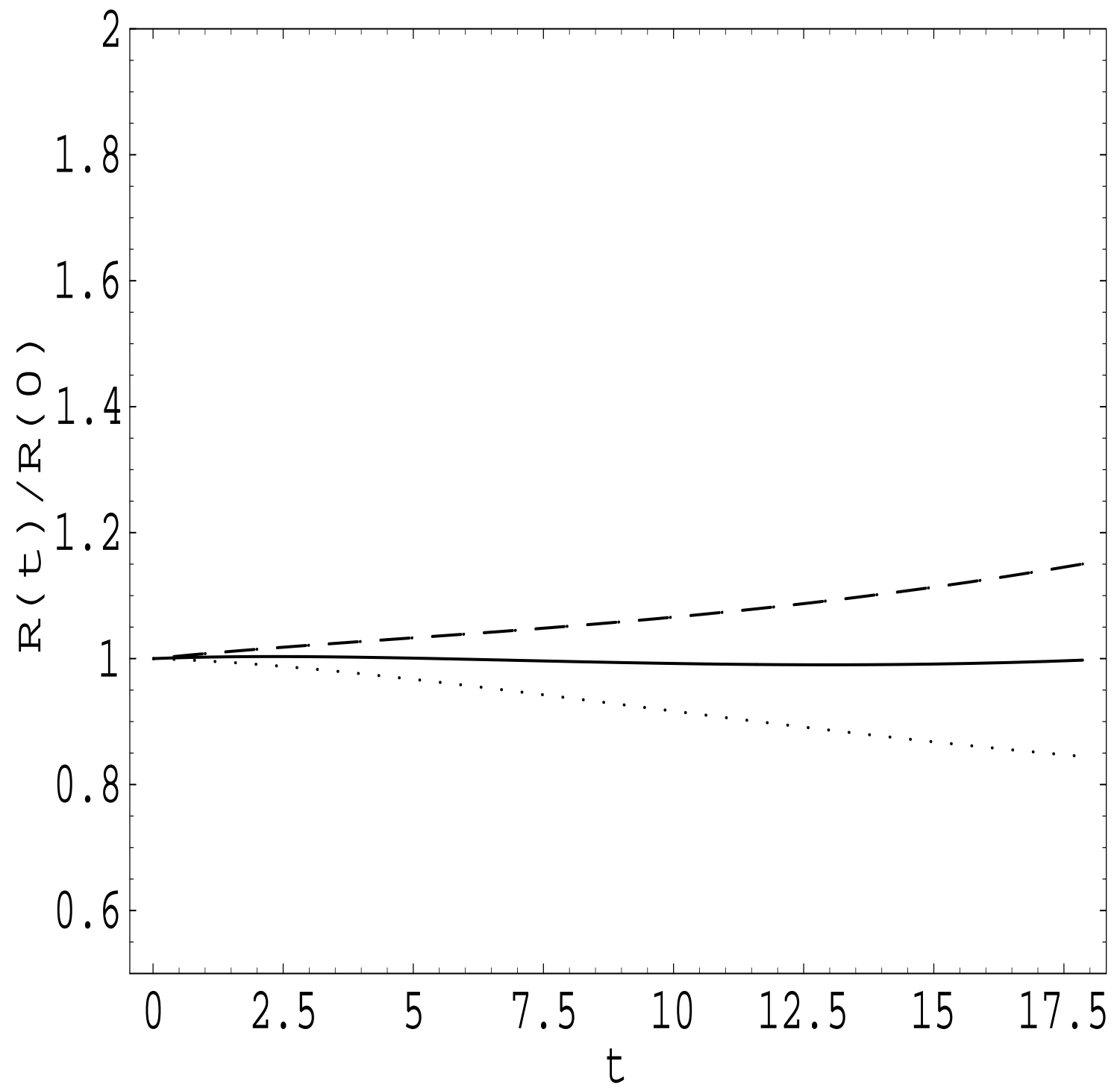

FIG. 2. Plot of $R(t) / R(0)$ vs. $t, R(t)=\lambda(t) / g_{t}^{2}(t), t=\ln \left(\mu / m_{t}\right)$ at one-loop, with $\lambda(0)=0.171,0.176,0.181, g_{t}(0)=0.95$ (dotted, solid and dashed respectively). 


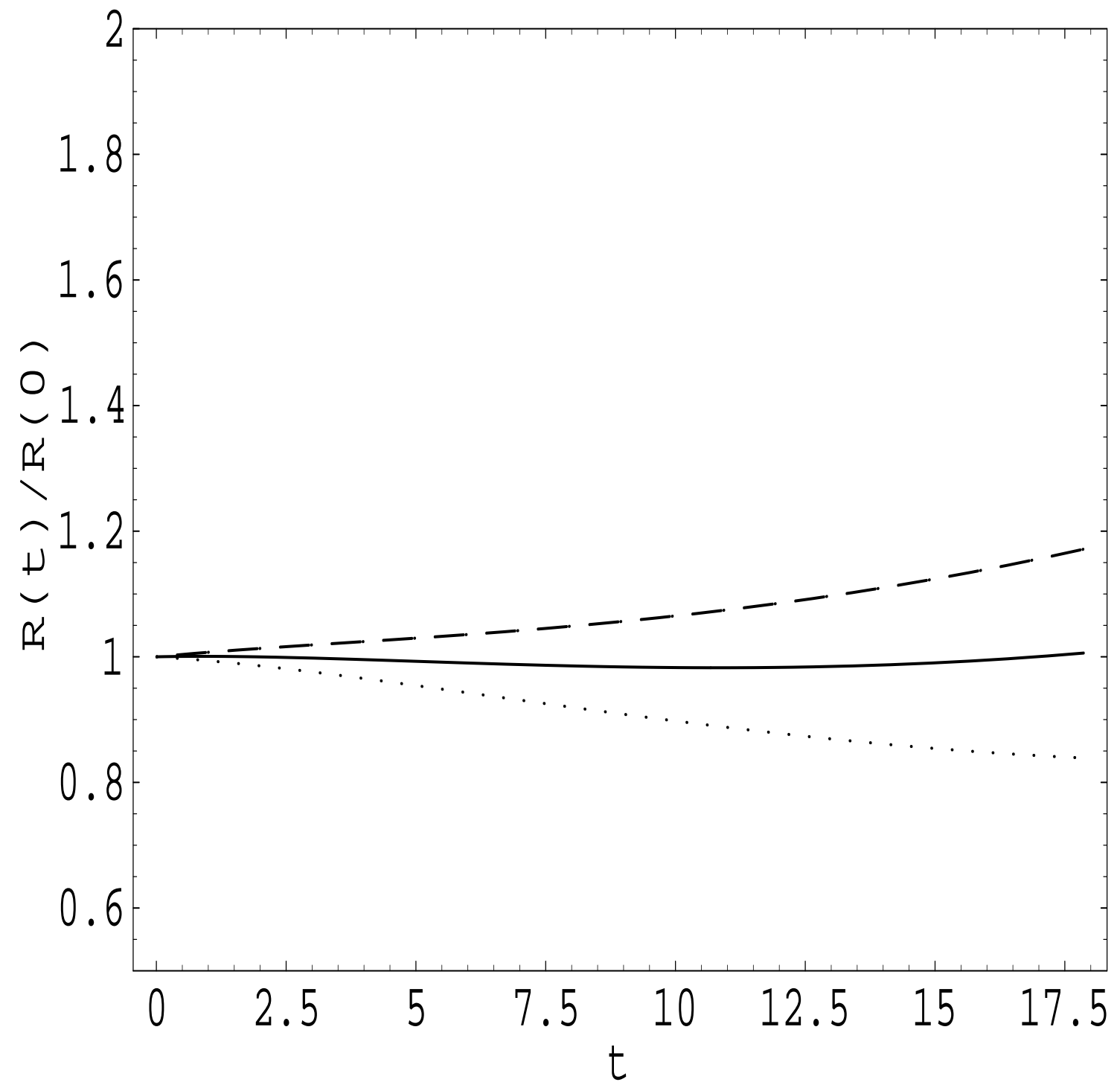

FIG. 3. Plot of $R(t) / R(0)$ vs. $t, R(t)=\lambda(t) / g_{t}^{2}(t), t=\ln \left(\mu / m_{t}\right)$ at two-loops, with $\lambda(0)=0.157,0.162,0.167, g_{t}(0)=0.95$ (dotted, solid and dashed respectively). 


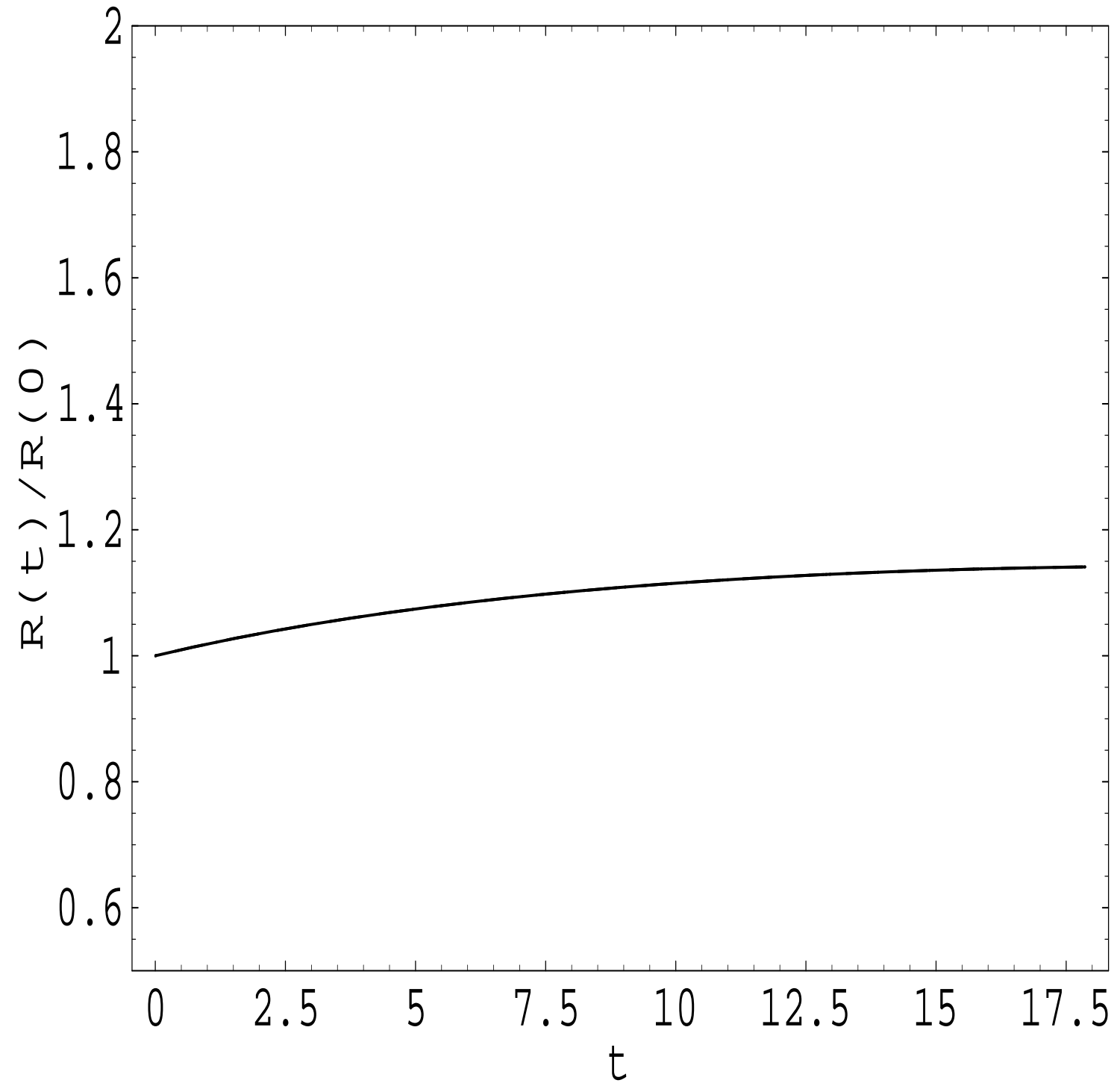

FIG. 4. Plot of $R(t) / R(0)$ vs. $t, R(t)=g_{3}^{2}(t) / g_{t}^{2}(t), t=\ln \left(\mu / m_{t}\right)$ at one-loop, with $g_{t}(0)=0.95$ (at one-loop the equations are independent of $\lambda$ ). 


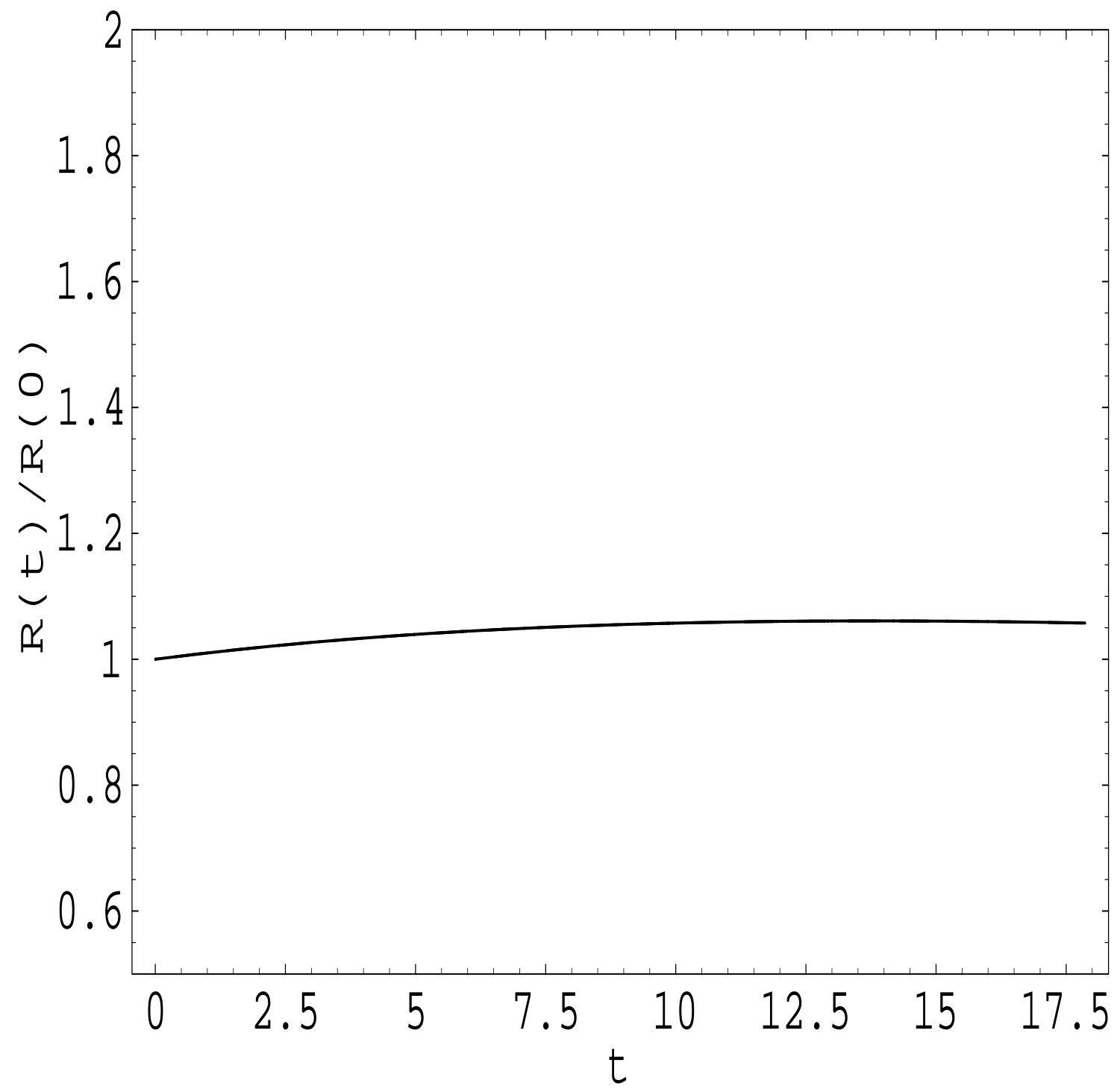

FIG. 5. Plot of $R(t) / R(0)$ vs. $t, R(t)=g_{3}^{2}(t) / g_{t}^{2}(t), t=\ln \left(\mu / m_{t}\right)$ at two-loops, with $g_{t}(0)=0.95$ and $\lambda(0)=0.162$. Varying $\lambda(0)$ to 0.157 or 0.167 has insignificant effect on this ratio, and the corresponding curves practically lie on top of the curve in the figure. 


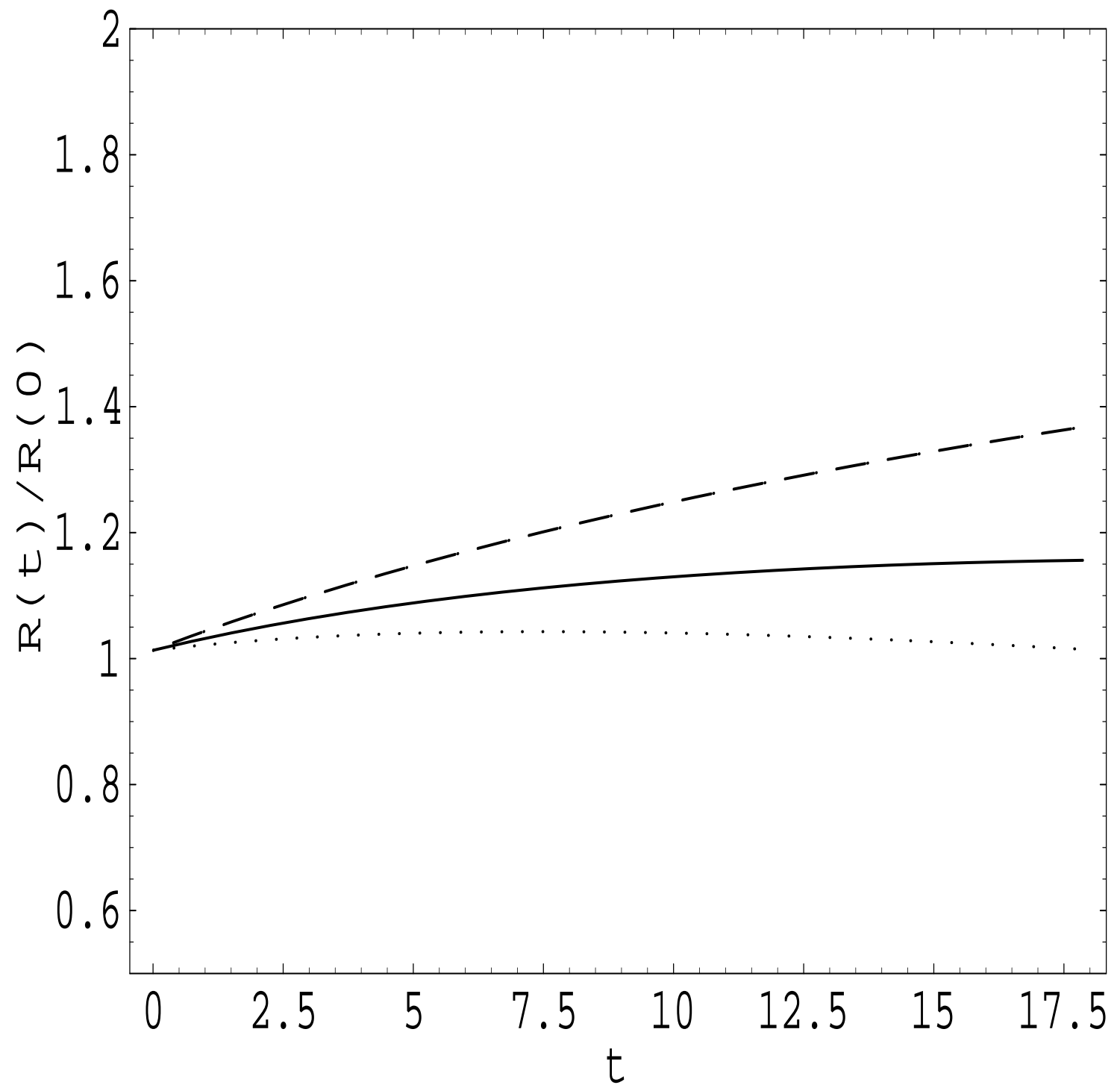

FIG. 6. Plot of $R(t) / R(0)$ vs. $t, R(t)=g_{3}^{2}(t) / g_{t}^{2}(t), t=\ln \left(\mu / m_{t}\right)$ at one-loop, with $g_{t}(0)=0.85,0.95,1.05$ (dotted, solid and dashed respectively). 


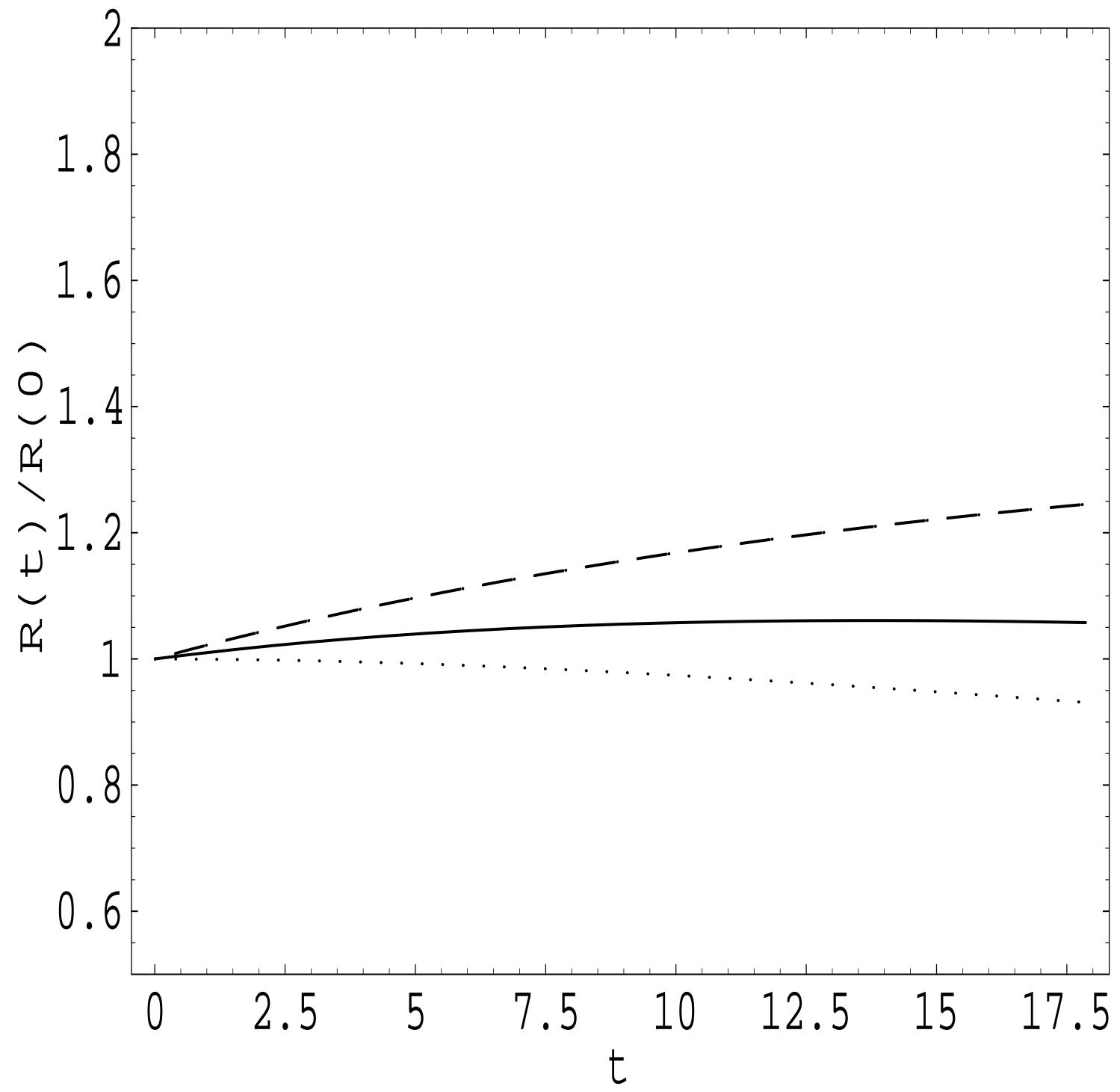

FIG. 7. Plot of $R(t) / R(0)$ vs. $t, R(t)=g_{3}^{2}(t) / g_{t}^{2}(t), t=\ln \left(\mu / m_{t}\right)$ at two-loops, with $\lambda(0)=0.162, g_{t}(0)=0.85,0.95,1.05$ (dotted, solid and dashed respectively). 